\title{
Temperature and filling dependence of the superconducting $\pi$-phase in the Penson-Kolb-Hubbard model
}

\author{
Fabrizio Dolcini* ${ }^{*}$ and Arianna Montorsi ${ }^{\dagger}$ \\ Dipartimento di Fisica and Unità INFM, Politecnico di Torino, I-10129 Torino, Italy
}

(November 7, 2017)

\begin{abstract}
We investigate in the Hartree Fock approximation the temperature and filling dependence of the superconducting $\pi$-phase for the PensonKolb-Hubbard model. Due to the presence of the pair-hopping term, the phase survives for repulsive values of the on-site Coulomb interaction, exhibiting an interesting filling and temperature dependence. The structure of the self-consistent equations peculiar to the $\pi$-phase of the model allows to explicitly solve them for the chemical potential. The phase diagrams are shown and discussed in dimension 2 and 3. We also show that, when a next-nearest neighbours hopping term is included, the critical temperature of the superconducting region increases, and the corresponding range of filling values is shifted away from half-filling. Comparison with known exact results is also discussed.
\end{abstract}

*e-mail: fdolcini@athena.polito.it

${ }^{\dagger}$ e-mail:montorsi@athena.polito.it 


\section{INTRODUCTION}

Interest in strongly correlated electron systems and superconductivity has motivated some attention on the wide class of Extended Hubbard models [1]. For such models, a certain number of exact results has been obtained for specific values of the interaction parameters [2,3], expecially in the one-dimensional case [4]- [9]. These results support in particular the existence of a superconducting phase at $T=0$, which is worth investigating also when relaxing some of the constraints among the parameters that allow the integrability of the Hamiltonian.

The Extended Hubbard Hamiltonian reads

$$
H_{E H}=H_{H u b}+H_{X}+H_{\tilde{X}}+H_{V}+H_{W}+H_{Y}
$$

where

$$
\begin{aligned}
H_{H u b} & =-t \sum_{<\mathbf{i}, \mathbf{j}>} \sum_{\sigma} c_{\mathbf{i}, \sigma}^{\dagger} c_{\mathbf{j}, \sigma}+U \sum_{\mathbf{i}} n_{\mathbf{i}, \uparrow} n_{\mathbf{i}, \downarrow} & & \text { (Hubbard) } \\
H_{X} & =X \sum_{<\mathbf{i}, \mathbf{j}>} \sum_{\sigma}\left(n_{\mathbf{i},-\sigma}+n_{\mathbf{j},-\sigma}\right) c_{\mathbf{i}, \sigma}^{\dagger} c_{\mathbf{j}, \sigma} & & \text { (bond-charge) } \\
H_{\tilde{X}} & =\tilde{X} \sum_{<\mathbf{i}, \mathbf{j}>} \sum_{\sigma} n_{\mathbf{i},-\sigma} n_{\mathbf{j},-\sigma} c_{\mathbf{i}, \sigma}^{\dagger} c_{\mathbf{j}, \sigma} & & \text { (correl. hopping } \leftrightarrow \text { on-site number) } \\
H_{V} & =\frac{V}{2} \sum_{<\mathbf{i}, \mathbf{j}>} n_{\mathbf{i}} n_{\mathbf{j}} & & \text { (neighboring site charge) } \\
H_{W} & =\frac{W}{2} \sum_{<\mathbf{i}, \mathbf{j}>} \sum_{\sigma, \sigma^{\prime}} c_{\mathbf{i}, \sigma}^{\dagger} c_{\mathbf{j}, \sigma^{\prime}}^{\dagger} c_{\mathbf{i}, \sigma^{\prime}} c_{\mathbf{j}, \sigma} & & \text { (exchange) } \\
H_{Y} & =Y \sum_{<\mathbf{i}, \mathbf{j}>} c_{\mathbf{i}, \uparrow}^{\dagger} c_{\mathbf{i}, \downarrow}^{\dagger} c_{\mathbf{j}, \downarrow} c_{\mathbf{j}, \uparrow} & & \text { (pair hopping) } .
\end{aligned}
$$

Here $c_{\mathbf{i}, \sigma}^{\dagger}$ and $c_{\mathbf{i}, \sigma}$ are fermionic creation and annihilation operators, the subscript $\mathbf{i}$ running over the sites of a $d$-dimensional lattice $\Lambda$ of $L^{d}$ sites, and $\sigma \in\{\uparrow, \downarrow\}$ being the spin label; the usual anticommutation rules $\left\{c_{\mathbf{i}, \sigma^{\prime}}, c_{\mathbf{j}, \sigma}\right\}=0$, $\left\{c_{\mathbf{i}, \sigma}, c_{\mathbf{j}, \sigma^{\prime}}^{\dagger}\right\}=\delta_{\mathbf{i}, \mathbf{j}} \delta_{\sigma, \sigma^{\prime}}$ hold. The symbol $<\mathbf{i}, \mathbf{j}>$ stands for nearest neighbors in $\Lambda$. Finally, $n_{\mathbf{i}, \sigma}=c_{\mathbf{i}, \sigma}^{\dagger} c_{\mathbf{i}, \sigma}$ is the electron number operator with spin $\sigma$ at site $\mathbf{i}$, and $n_{\mathbf{i}}=n_{\mathbf{i} \uparrow}+n_{\mathbf{i} \downarrow}$.

As Hubbard himself pointed out in [1], all the terms in (四) appear due to the second 
quantization formulation of the interaction among electrons. The specific physical system of interest then suggests which are the most relevant ones.

For the pure Hubbard model no exact result proves the existence of a superconducting phase at finite values of $U$, and even within a mean-field scheme this is achieved only for negative $U$ values. On the contrary a number of interesting exact results on the existence of a superconducting phase for appropriate non-vanishing values of some other interaction parameters in (11) has been obtained. Most of these results involve the notion of the states known as $\eta_{\phi^{-}}$-pairs, i.e. the states

$$
|\eta\rangle_{\phi}=\left(K_{\phi}^{\dagger}\right)^{m}|0\rangle \quad, \quad m=1, \ldots, L^{d}
$$

with

$$
K_{\phi}^{\dagger}=\sum_{\mathbf{j} \in \Lambda} e^{i \boldsymbol{\phi} \cdot \mathbf{j}} c_{\mathbf{j} \uparrow}^{\dagger} c_{\mathbf{j} \downarrow}^{\dagger}=\sum_{\mathbf{k} \in B} c_{\boldsymbol{\phi}-\mathbf{k} \downarrow}^{\dagger} c_{\mathbf{k} \uparrow}^{\dagger}
$$

where $\phi$ is a $d$-dimensional vector $(\phi, \phi, \ldots)$, and $B$ is the first Brillouin zone in the reciprocal lattice. Noticeably, the states $|\eta\rangle_{\phi}$ enjoy the property of 'Off Diagonal Long Range Order' (ODLRO), which implies superconductivity [10]. Therefore much effort has been done through the last years to find which are the relations among the coupling parameters in (1) guaranteeing that an $|\eta\rangle_{\phi}$ is the ground state. A first set of remarkable results in 1-d was obtained for the subclass of Hamiltonians characterized by the constraint $X=t$. In [6, [] the phase diagram $U$ vs filling for the AAS model $X=t, \tilde{X}=W=V=Y=0$ (reported in fig.四) was derived at $T=0$ : one can see a superconductive filling-independent region, where the $|\eta\rangle_{\phi}$ are degenerate ground states for any $\phi$, and a filling dependent zone (again superconducting because it contains at least $|\eta\rangle_{0}$ pairs) rising up to positive values of $U$. Unfortunately, in contrast to the real case of superconducting materials, the superconducting phase turns out to have a maximum at half filling. A similar phase diagram (see fig. 1) was also obtained in 4,5 for the EKS Hamiltonian, 
characterized by $X=t, \tilde{X}=0, Y=W=V=-1$. There the filling independent phase is made of $|\eta\rangle_{\phi}$ with just $\phi=0$, since $Y \neq 0$. In fact a non-vanishing pairhopping term removes the degeneracy of $|\eta\rangle_{\phi},|\eta\rangle_{0}$ being energetically favourite for $Y<0$, while $|\eta\rangle_{\pi}$ is favourite for $Y>0$. Moreover, as fig.1 shows, a non-vanishing $Y$ also contributes to rise up the superconducting region towards positive values of U.

More recently, it has also been realized [3] that, at least in order to obtain a filling independent superconducting ground state region, some of the constraints on the parameters (in particular $X=t$, which is not very physical) are not necessary, provided that a pair-hopping term is present $(Y \neq 0)$. Notice that, as soon as $X \neq t$, only $|\eta\rangle_{\pi}$ states could become ground states, the other choices of $\phi$ in (2) giving states that cannot be eigenstates of (11).

On the contrary, no exact result is available concerning the existence of the more structured filling dependent superconducting region when the constraints on the parameters which allow the 1- $d$ integrability are removed. It is one purpose of the present paper to investigate within the Hartree-Fock scheme such possibility, as well as to test how the superconducting region modifies at $T \neq 0$.

Given the relevance of the pair-hopping term to stabilize the $\eta_{\pi}$-pairs phase, and in order to make the physical mechanism more clear, we shall focus on a subcase of the extended model in which, apart from the pure Hubbard terms, only the pair hopping amplitude is taken different from zero. This is known in the literature as Penson-Kolb-Hubbard model. We want to emphasize here that the presence of other terms in (11) is not expected to affect our results in a qualitative way, as other recent numerical studies confirm [11, 12].

In Section $\llbracket$ we give the Hamiltonian and derive within the Hartree-Fock scheme the temperature dependent equations for the filling and the self-consistent supercon- 
ducting order parameter. In Section III we solve the equations in dimension 2 and 3, and show the temperature and filling dependence of the superconducting phase in these cases. In Section IV we add to the Hamiltonian a next-nearest neighbors contribution to the hopping term, and we show how this affects the filling and temperature dependence of the superconducting phase. Finally, in Section $\square$ we discuss our results and give some conclusions.

\section{PENSON-KOLB-HUBBARD MODEL AND HARTREE-FOCK (HF) APPROACH TO THE $\pi$-PHASE}

The Penson-Kolb-Hubbard Hamiltonian reads

$$
H_{P K H}=-t \sum_{<\mathbf{i}, \mathbf{j}>} \sum_{\sigma} c_{\mathbf{i}, \sigma}^{\dagger} c_{\mathbf{j}, \sigma}+U \sum_{\mathbf{i}} n_{\mathbf{i}, \uparrow} n_{\mathbf{i}, \downarrow}+Y \sum_{<\mathbf{i}, \mathbf{j}>} c_{\mathbf{i}, \uparrow}^{\dagger} c_{\mathbf{i}, \downarrow}^{\dagger} c_{\mathbf{j}, \downarrow} c_{\mathbf{j}, \uparrow}+\mu \sum_{\mathbf{i}} n_{\mathbf{i}}
$$

where the last term accounts for the chemical potential.

The case $U=0$ in 1- $d$ was first examined by Penson and Kolb [13] to study a short range interaction between electron pairs of small radius (actually zero), in contrast with the BCS-theory, where the size of the Cooper pairs is comparatively large. This led to envisage a real space formulation for the electron pairing, which is very interesting in many contexts of condensed matter physics.

Later on, the Coulomb repulsion term $U$ was also taken into account by [14, where the PKH Hamiltonian was proposed as an effective phenomenological model capturing the main physical features of doped materials, such as high $T_{c}$ superconductors. Indeed if we assume that, due to some (yet unknown) microscopic mechanism, localized pairs can be formed, then their displacement in the lattice should be described by a pair hopping term competiting with a single carrier hopping amplitude $t$. The Coulomb repulsion term should account for the insulating phase. 
More recently a slave boson wide study of the different possible phases of the model at zero temperature has also been done 15]. In particular, a region characterized by a non-vanishing value of the order parameter $x_{\pi}=\frac{1}{L^{d}}<K_{\pi}^{\dagger}>$ was found; here $K_{\pi}^{\dagger}$ is given by (3) with $\phi=\pi$, and $<>$ stands for the average value on the grand canonical statistical ensemble. In the following we shall denote such a phase as $\pi$-phase.

Noticing that $H_{Y}$ in (1) can be rewritten also as $-Y \sum_{<\mathbf{i}, \mathbf{j}>} e^{i \boldsymbol{\pi} \cdot(\mathbf{i}-\mathbf{j})} c_{\mathbf{i}, \uparrow}^{\dagger} c_{\mathbf{i}, \downarrow}^{\dagger} c_{\mathbf{j}, \downarrow} c_{\mathbf{j}, \uparrow}$, and assuming a translational invariance throughout the lattice, a Hartree-Fock linearization can be implemented on both interaction terms in (4) to study the $\pi$-phase. We shall suppose that such a phase is the energetically favourite one; therefore we shall take $Y>0$ and assume that the thermal energy is not sufficient to let other phases emerge (see 15 for a detailed study of the possible several phases). Within this HF approximation the PKH Hamiltonian decomposes into a sum of $k$-space independent hamiltonians,

$$
H_{P K H} \stackrel{H F}{\approx} \sum_{\mathbf{k} \in B} H_{\mathbf{k}} \quad, \quad\left[H_{\mathbf{k}}, H_{\mathbf{k}^{\prime}}\right]=0
$$

where

$H_{\mathbf{k}}=-\left[\left(t \epsilon_{\mathbf{k}}+\mu\right) n_{\mathbf{k} \uparrow}+(t \epsilon \boldsymbol{\pi}-\mathbf{k}+\mu) n \boldsymbol{\pi}-\mathbf{k} \downarrow\right]+\tilde{U}\left(x_{\pi} c_{\mathbf{k} \uparrow}^{\dagger} c^{\dagger} \boldsymbol{\pi}-\mathbf{k} \downarrow_{1}+x_{\pi}^{*} c \boldsymbol{\pi}-\mathbf{k} \downarrow c_{\mathbf{k} \uparrow}\right)-\tilde{U}\left|x_{\pi}\right|^{2}$

and $\tilde{U}=U-q Y, q$ being the number of nearest neighbors, equal to $2 d$ for a hypercubic lattice. The sum in (5) runs over the Brillouin zone $B$ and the $\mathbf{k}$ vectors are measured in units of the inverse lattice spacing (i.e. $\left.-\pi \leq k_{i} \leq \pi\right)$.

In contrast with (4), the linearized hamiltonian (6) does not preserve the number of particles; indeed in a HF picture the $\pi$-phase has to be thought of as a superposition of $\eta_{\pi}$-pairs involving different number of pairs, the average number of electrons being fixed through the chemical potential $\mu$. 
Since the dynamical algebra of each $H_{\mathbf{k}}$ is the $S U(2) \oplus U(1)$ given by the following generators:

$$
\begin{aligned}
& J_{\mathbf{k}}^{\dagger}=c_{\mathbf{k} \uparrow}^{\dagger} c^{\dagger} \boldsymbol{\pi}-\mathbf{k} \downarrow \\
& J_{\mathbf{k}}^{-}=c \boldsymbol{\pi}_{-\mathbf{k} \downarrow} c_{\mathbf{k} \uparrow} \\
& J_{\mathbf{k}}^{z}=(n \boldsymbol{\pi}-\mathbf{k} \uparrow+n \boldsymbol{\pi}-\mathbf{k} \downarrow-1) / 2 \\
& S_{\mathbf{k}}^{z}=(n \boldsymbol{\pi}-\mathbf{k} \uparrow-n \boldsymbol{\pi}-\mathbf{k} \downarrow) / 2,
\end{aligned}
$$

we can diagonalize it by means of the following unitary transformation,

$$
H_{\mathbf{k}} \rightarrow H_{\mathbf{k}}^{\prime}=e^{L_{\mathbf{k}}} H_{\mathbf{k}} e^{-L_{\mathbf{k}}} \quad, \quad L_{\mathbf{k}}=a_{\mathbf{k}}\left(x_{\pi} J_{\mathbf{k}}^{\dagger}-x_{\pi}^{*} J_{\mathbf{k}}^{-}\right)
$$

where $a_{\mathbf{k}}$ are real numbers satisfying the relation

$$
\tan \left(2 a_{\mathbf{k}}\left|x_{\pi}\right|\right)=-\frac{\left|x_{\pi}\right| \tilde{U}}{\mu+t\left(\epsilon_{\mathbf{k}}+\epsilon \boldsymbol{\pi}-\mathbf{k}\right) / 2} .
$$

The grand partition function $Z$ in the HF approximation can then be worked out, since

$$
Z=\operatorname{Tr} e^{-\beta H_{P K H}} \stackrel{H F}{\approx} \prod_{\mathbf{k}} \operatorname{Tr}_{\mathbf{k}} e^{-\beta H_{\mathbf{k}}}=\prod_{\mathbf{k}} \operatorname{Tr}_{\mathbf{k}} e^{-\beta H_{\mathbf{k}}^{\prime}}
$$

where $T r_{\mathbf{k}}$ is the trace over the space of quantum labels $(\mathbf{k}, \uparrow)$ and $(\boldsymbol{\pi}-\mathbf{k}, \downarrow)$.

The thermodynamic grand potential (per particle) in the thermodynamic limit now reads

$$
\begin{aligned}
& \omega=\lim _{T . D .}-k_{B} T \frac{\ln Z}{N}= \\
& =-\frac{1}{(2 \pi)^{d}} \int_{B} d \mathbf{k}\left[A_{\mathbf{k}}+k_{B} T \ln \left[2 \cosh \beta \frac{D_{\mathbf{k}}+R_{\mathbf{k}}}{2}\right]+\ln \left[2 \cosh \beta \frac{D_{\mathbf{k}}-R_{\mathbf{k}}}{2}\right]\right]
\end{aligned}
$$

with

$$
\begin{gathered}
S_{\mathbf{k}}=t\left(\epsilon_{\mathbf{k}}+\epsilon \boldsymbol{\pi}-\mathbf{k}\right) / 2 \\
D_{\mathbf{k}}=t\left(\epsilon_{\mathbf{k}}-\epsilon \boldsymbol{\pi}-\mathbf{k}\right) / 2 \\
A_{\mathbf{k}}=\mu+S_{\mathbf{k}}+\tilde{U}\left|x_{\pi}\right|^{2} \\
R_{\mathbf{k}}=\sqrt{\left(\mu+S_{\mathbf{k}}\right)^{2}+\tilde{U}^{2}\left|x_{\pi}\right|^{2}} .
\end{gathered}
$$


In order to investigate the thermodynamical properties of the system one has to implement the selfconsistency equation $\partial \omega / \partial x_{\pi}=0$ for the order parameter, which turns out to be

$$
x_{\pi} \cdot \int_{B} d \mathbf{k}\left[1+\frac{\tilde{U}}{4 R_{\mathbf{k}}}\left[\tanh \beta \frac{D_{\mathbf{k}}+R_{\mathbf{k}}}{2}-\tanh \beta \frac{D_{\mathbf{k}}-R_{\mathbf{k}}}{2}\right]\right]=0 .
$$

As usual, this equation gives a solution $x_{\pi} \equiv 0$ for $T \geq T_{c}$ and a solution $x_{\pi} \neq 0$ for $T \leq T_{c}$. It is easy to see that the $\pi$-phase exists only when $\tilde{U}=U-q Y \leq 0$, that is when the pair-hopping $Y>0$ term renormalizes the effective interaction $U>0$ to an attractive regime. Indeed for $T \leq T_{c}$ the above equation gives:

$$
\tilde{U}^{-1}=-\frac{1}{(2 \pi)^{d}} \int_{B} d \mathbf{k} \frac{1}{4 R_{\mathbf{k}}}\left[\left[\tanh \beta \frac{D_{\mathbf{k}}+R_{\mathbf{k}}}{2}-\tanh \beta \frac{D_{\mathbf{k}}-R_{\mathbf{k}}}{2}\right]\right]=0 .
$$

Moreover, one must also satisfy the filling equation, $n=\partial \omega / \partial \mu$, which reads

$$
n=1+\frac{1}{(2 \pi)^{d}} \int_{B} d \mathbf{k} \frac{\mu+S_{\mathbf{k}}}{2 R_{\mathbf{k}}}\left[\tanh \beta \frac{D_{\mathbf{k}}+R_{\mathbf{k}}}{2}-\tanh \beta \frac{D_{\mathbf{k}}-R_{\mathbf{k}}}{2}\right] .
$$

Eqns. (9)-(10) constitute the parametric form of the equation of state. In order to get to one closed form, one should in principle invert (10) obtaining $\mu$ as a function of $n, T$ and $\tilde{U}$, and then insert it into (9). The thermodynamics of the model will then be expressed in terms of $n, T$ and $\tilde{U}$.

Noticeably, comparing (9) and (10) it is easy to show that whenever $S_{\mathbf{k}}$ in (8) vanishes, i.e. whenever:

$$
\epsilon \boldsymbol{\pi}-\mathbf{k}=-\epsilon_{\mathbf{k}}
$$

the chemical potential can be exactly inverted. In this case we simply have:

$$
\mu=\frac{1-n}{2} \tilde{U}
$$

We wish to stress here that for a given model, even within the HF approximation, it is not obvious at all that $\mu$ can be inverted exactly: in the $\mathrm{PKH}$ model it is a 
peculiar feature of the $\pi$-phase (which is not shared by $|\eta\rangle_{0}$ phase).

The relations (11)-(12) hold in any dimension for a hypercubic lattice when dealing with a nearest neighbors hopping term, since the dispersion relations reads:

$$
\epsilon_{\mathbf{k}}=\sum_{i=1}^{d} 2 \cos k_{i}
$$

As we will discuss in IV, eqn.(11) is no more satisfied when a next nearest neighbors term is included.

\section{THE TEMPERATURE AND FILLING DEPENDENCE OF THE PHASE DIAGRAM IN $D=2$ AND $D=3$}

In this section we consider the case of a nearest neighbors hopping term, the dispersion relation being given by (13). We aim to derive the features of the critical values of $\tilde{U}$ versus $n$ for a given temperature $T$, in order to compare them with

known solution of similar models (see section $\mathrm{\nabla}$ ). Such a critical curve $\tilde{U}_{c}=\tilde{U}_{c}(n)$ is obtained from (9)-(10) by setting the order parameter $x_{\pi}=0$ into (8).

As already noticed in section 【1, besides the parametric form (9)-(10), in this case we can also deal with one closed form; indeed, since (11) is satisfied, $R_{\mathrm{k}}$ is independent of $\mathbf{k}$, and therefore (12) holds. By substituting $\mu$ into $R_{\mathbf{k}}$, and then $R_{\mathbf{k}}$ into (9), we obtain from (10) the critical equation

$$
\frac{1}{(2 \pi)^{d}} \int_{B} d \mathbf{k} \frac{1}{2}\left[\tanh \left[\beta \frac{t \epsilon_{\mathbf{k}}+\tilde{U}_{c} \delta(n) / 2}{2}\right]-\tanh \left[\beta \frac{t \epsilon_{\mathbf{k}}-\tilde{U}_{c} \delta(n) / 2}{2}\right],\right]=\delta(n)
$$

where $\delta(n)=1-n$ is the 'doping' and $B$ is the $d$-dimensional Brillouin zone.

In order to work out the integration it is worth to introduce the 'density of states' $g^{(d)}(\epsilon)$ with normalization

$$
\frac{1}{(2 \pi)^{d}} \int_{B} d \mathbf{k}=\int_{\epsilon_{\min }}^{\epsilon_{\max }} d \epsilon g^{(d)}(\epsilon)
$$


which turns out to be in this case an even function of $\epsilon$, so that $\epsilon_{\min }=-\epsilon_{\max }$ (this will not be the case in section $[\mathrm{IV})$. Moreover we have $\epsilon_{\max }=2 d$.

In $d=2$ it is known [16] that

$$
g^{(2)}(\epsilon)=\frac{1}{2 \pi^{2}} K\left(1-\left(\frac{\epsilon}{4}\right)^{2}\right)
$$

where $K$ is the complete elliptic integral of the first kind; this function has a logarithmic divergence at $\epsilon=0$.

For the three-dimensional case we have also performed the calculation, obtaining

$$
g^{(3)}(\epsilon):=\begin{array}{ll}
\frac{2}{\pi} \int_{0}^{\pi} g^{(2)}(\epsilon / 2-\cos (x)) d x & 0 \leq|\epsilon| \leq 2 \\
\frac{2}{\pi} \int_{0}^{\arccos \left(\frac{\epsilon}{2}-2\right)} g^{(2)}(\epsilon / 2-\cos (x)) d x & 2 \leq|\epsilon| \leq 6,
\end{array}
$$

which is plotted in fig.2.

Since eqn.(14) is invariant under the transformation $\delta(n) \rightarrow-\delta(n)$, it is easily seen that $\tilde{U}_{c}$ is symmetric with respect to the value at half filling $(n=1)$. The critical curves in $d=2$ and $d=3$ are plotted in figs.3 and 14 respectively. For the sake of consistency with our approximation, we have plotted the region of the phase diagram where the values of $\tilde{U}$ do not exceed the band-width $\Delta=4 t d$.

Notice the different behaviour of $\tilde{U}_{c}$ in the two cases, in particular for low temperatures. Indeed in $d=2$ we have a very sharp, cuspid-like shape at half filling, while in $d=3$ a 'plateau' is obtained, meaning that the effective interaction threshold is almost independent of the density of electrons in the lattice for a rather wide range of $n$. This effect is due to the quite different behaviour of the density of states $g^{(2)}$ and $g^{(3)}$. Indeed using the parametric form it is possible to show that at low $T$ 's the shape of $\tilde{U}_{c}$ in the neighborhood of half filling is governed by the behaviour of $g^{(d)}$ around $\epsilon=0$. In fact from eqns. (12) and (14) we easily obtain that the value of $\mu$ is determined by the equation 


$$
\int_{-\epsilon_{\max }}^{+\epsilon_{\max }} g^{(d)}(\epsilon) P_{\beta}(\epsilon ; \mu)=1-n
$$

where $\beta=1 / k_{B} T$, and $P_{\beta}(\epsilon ; \mu)$ is

$$
P_{\beta}(\epsilon ; \mu)=\frac{1}{2}\left[\tanh \beta t \frac{\epsilon-\mu}{2}-\tanh \beta t \frac{\epsilon+\mu}{2}\right] .
$$

Taking into account the normalization (15) of $g^{(d)}(\epsilon)$ and the fact that $P_{\beta}(\epsilon ; \mu)$ is positive and not greater than 1 , one can easily see that $n=1$ implies $\mu=0$ (for any temperature $T$ ). Since from eqn.(12) we have $\tilde{U}=2 \mu /(1-n)$, a simple limit $\mu \rightarrow 0$ can be performed, yielding

$$
\tilde{U}_{c}(n=1)=-\left(\int_{-\epsilon_{\max }}^{+\epsilon_{\max }} g^{(d)}(\epsilon) p_{\beta}(\epsilon)\right)^{-1},
$$

where

$$
p_{\beta}(\epsilon)=\frac{\beta t}{4} \cosh ^{-2}\left(\frac{\beta t}{2} \epsilon\right)
$$

This holds for any temperature $T$. In particular when $T \rightarrow 0$ one gets

$$
\tilde{U}_{c}(n=1)=-\frac{1}{g^{(d)}(0)}
$$

Now, since $g^{(3)}$ is almost constant around $\epsilon=0$, when $T \sim 0$ eqn. (18) gives

$$
1-n \approx \int_{-|\mu|}^{+|\mu|} g^{(3)}(\epsilon) \approx-\left.2 \mu g^{(3)}(\epsilon)\right|_{\epsilon=0} \quad,
$$

and using (12) we obtain that $\tilde{U}_{c}$ is actually independent of $n$ (indeed $g^{(3)}(0)=$ 0.1427 and so $1 / 0.1427=7.0078$ which is just the value of $\tilde{U}$ around half filling when $T=0$ ). To be more precise, since the value of $g^{(3)}$ at $|\epsilon|=2$ is slightly higher than at $\epsilon=0$ (see fig.21), we have that at $T=0$ the plateau is slightly concave, so that the highest value of $\tilde{U}_{c}$ is actually reached away from half filling, at the symmetric values $n \sim 0.4$ and $n \sim 1.6$.

Eqn.(21) stems from eqn.(19) because the functions $p_{\beta}(\epsilon)$ are 'regularizing' in the 
sense that they are positive and their integral on $\mathcal{R}$ equals 1 for any $\beta$ (though strictly speaking they belong to $\mathcal{S}(\mathcal{R})$ and not to $\mathcal{C}_{0}^{\infty}(\mathcal{R})$ ). In fact in $d=2$ we observe that, when $\beta \rightarrow \infty$,

$$
\begin{aligned}
\int_{-4}^{4} g^{(2)}(\epsilon) p_{\beta}(\epsilon) \geq \int_{-2 /(\beta t)}^{2 /(\beta t)} & g^{(2)}(\epsilon) p_{\beta}(\epsilon) \geq \\
& \geq \underbrace{\int_{-2 /(\beta t)}^{2 /(\beta t)} p_{\beta}(\epsilon)}_{2 \tanh 1} \cdot \inf _{0 \leq|\epsilon| \leq 2 /(\beta t)} g^{(2) \stackrel{\beta \rightarrow \infty}{\longrightarrow} \infty,}
\end{aligned}
$$

because of the divergence of $g^{(2)}$ at $\epsilon=0$.

The $3-d$ case is slightly different because $g^{(3)}$ is finite at $\epsilon=0$; nevertheless, if we take a sufficiently small $\delta$ around $\epsilon=0$, it easily realized that

$$
\lim _{\beta \rightarrow \infty} \int_{\delta \leq|\epsilon| \leq \epsilon_{\max }} g^{(3)}(\epsilon) p_{\beta}(\epsilon) d \epsilon=0=\lim _{\beta \rightarrow \infty} \int_{\delta \leq|\epsilon| \leq \epsilon_{\max }} p_{\beta}(\epsilon) d \epsilon
$$

which yields

$$
\inf _{|\epsilon| \leq \delta} g^{(3)} \leq \lim _{\beta \rightarrow \infty} \int_{-\epsilon_{\max }}^{+\epsilon_{\max }} g^{(3)}(\epsilon) p_{\beta}(\epsilon) \leq \sup _{|\epsilon| \leq \delta} g^{(3)} \quad \forall \delta>0
$$

and so again (21).

\section{THE NEXT NEAREST NEIGHBORS CONTRIBUTION}

Let us now turn to the case when in the Hamiltonian (₫) a next nearest neighbors contribution is included in the hopping term, which therefore becomes

$$
-t \sum_{<\mathbf{i}, \mathbf{j}>} \sum_{\sigma} c_{\mathbf{i}, \sigma}^{\dagger} c_{\mathbf{j}, \sigma}-\alpha t \sum_{<<\mathbf{i}, \mathbf{j}>>} \sum_{\sigma} c_{\mathbf{i}, \sigma}^{\dagger} c_{\mathbf{j}, \sigma}
$$

Notice that such a term explicitly breaks the particle-hole $c_{\mathbf{j}}^{\dagger} \rightarrow e^{i \boldsymbol{\pi} \cdot \mathbf{j}} c_{\mathbf{j}}$ invariance of the model. The dispersion relation is now given by

$$
\epsilon_{\mathbf{k}}=\sum_{i=1}^{d} 2 \cos k_{i}+\alpha \prod_{i<j \leq d} 4 \cos k_{i} \cos k_{j}
$$


As observed at the end of section II, the symmetry $\epsilon_{\mathbf{k}}=-\epsilon_{\boldsymbol{\pi}-\mathbf{k}}$ does not hold anymore; as a consequence, $S_{\mathbf{k}}$ in eqn.(8) is no more vanishing, and $R_{\mathbf{k}}$ does depend on k. This yields both mathematical and physical new features. In particular, the equation for the critical surface has to be given in the parametric form (9)-(10), $\mu$ being the parameter.

We are here interested in the case $d=2$. We have computed also in this case the density of states, that turns out to be

$$
g_{\alpha}^{(2)}(\epsilon)=\frac{1}{2 \pi^{2} \sqrt{1+\alpha \epsilon}} K\left(\frac{1-(\epsilon / 4-\alpha)^{2}}{1+\alpha \epsilon}\right),
$$

where $\alpha$ is assumed to be $|\alpha| \leq 1 / 2$, since the next-nearest neighbors term is expected to be small (in $L^{2}$ ) with respect to the nearest neighbors one. For $\alpha=0$ we recover the usual form (16). Notice also that the function $g_{\alpha}^{(2)}$ is no more even in $\epsilon$; indeed we now have $g_{\alpha}^{(2)}(-\epsilon)=g_{-\alpha}^{(2)}(\epsilon)$. The logarithmic divergence is shifted to $\epsilon=-4 \alpha$, and the domain is characterized by $\epsilon_{\min }=-4(1-\alpha)$ and $\epsilon_{\max }=4(1+\alpha)$, as shown in fig. 5 .

This imparts the critical curve $\tilde{U}_{c}$ vs $n$ an asymmetric form, the highest value of $\tilde{U}$ falling now at a $n_{\max } \neq 1$, as shown in fig.6. Moreover, at a given temperature, such a maximum of the critical curve is shifted upward with respect to the curve of the case $\alpha=0$. This means that at a given temperature $T$, the effect of the next nearest neighbors term is to reduce the 'optimal' effective attraction $\tilde{U}$. In turn, this implies at a given $\tilde{U}$ the raising of the highest critical temperature reachable by doping the system.

We have also studied how $n_{\max }$ depends on $\alpha$. The relation is almost linear for $-0.4 \leq \alpha \leq 0.4$, while it displays a sudden increase around $\alpha \sim 0.45$; in fig. 0 we have extended the curve to the range $0 \leq|\alpha| \leq 1$ (which could be still acceptable in principle) to show how $n_{\max }$ saturates towards the limiting values $n=0$ or $n=2$. Notice that the curve is odd in $\alpha$; this is because the parametric eqns.(9)-(10) are 
invariant under the transformation $\mu \rightarrow-\mu ; \alpha \rightarrow-\alpha ; \tilde{U} \rightarrow \tilde{U}$, and therefore one can show, in agreement with [16], that

$$
T_{c}(\tilde{U}, n ; \alpha)=T_{c}(\tilde{U}, 2-n ;-\alpha)
$$

It is possible to see that the curve depends very weakly on the temperature $T$.

Finally, we have plotted the phase diagram of $T_{c}$ versus $n$ at fixed $\alpha$, which is shown in fig.8. We can observe that the next nearest neighbors term has mainly two effects. First it shifts away from half filling the range of values of $n$ at which the superconducting phase exists; this suggests that the system has to be doped in order to observe a superconducting behaviour. Secondly, it raises up the highest reachable critical temperature with respect to the case where only a nearest neighbors interaction is considered. Having in mind the phenomenology of high $T_{c}$ materials, this study supports the idea that the actual microscopic Hamiltonian should be particle-hole not-invariant.

\section{DISCUSSION AND CONCLUSIONS}

The phase diagrams obtained within the Hartree-Fock scheme can be given a more precise physical interpretation by comparing them with the 1-d exact results known for some very specific cases. Strictly speaking, such a comparison is only possible for $T=0$, since the phase diagram of these integrable models is not known at $T \neq 0$. In so doing, we observe that at zero temperature our results in fig.3 and fig. 4 have the same structure as those in fig.11. One can recognize three distinct regions in the phase space. The first one is characterized by filling independent $\pi$-phase. By reducing the effective attraction $\tilde{U}$, one enters a second region in which the existence of the $\pi$-phase depends on the actual filling. Finally, above the critical curve the $\pi$-phase disappears. 
In analogy with what is known to happen for the solved 1-d cases, we can think of the filling independent region as the phase in which all particles are paired in $\eta_{\pi}$-pairs (2). This is in agreement with known result in $d>1$ [3]. The second region should be characterized by simultaneous presence of paired and unpaired electrons and empty sites, whereas in the third one no paired electrons could move.

Switching on the temperature, thermal fluctuations are expected to break pairs. The dependence on the temperature of our phase diagrams supports this idea. Actually for a given filling $n$, the greater becomes the temperature $T$, the greater must be the magnitude of the effective attractive interaction $\tilde{U}$ in order to keep the $\eta_{\pi^{-}}$ pairs bound together. In fact this is what our phase diagrams in figs. 3 and 1 show: the curves of higher T's lay below the lower T's ones; this can also be demonstrated analitically very easily, at least in the cases of a nearest neighbors hopping term. Indeed from eqn.(14), which is of the form $G\left(n, \beta, \tilde{U}_{c}\right)=1-n$, we obtain that

$$
\frac{d \tilde{U}_{c}}{d T}=-\frac{1}{k_{B} T^{2}} \frac{d \tilde{U}_{c}}{d \beta}=-\frac{1}{k_{B} T^{2}} \frac{\partial G / \partial \beta}{\partial G / \partial \tilde{U}_{c}}
$$

By working out the derivatives, and using the fact that the functions $\tanh (x)$ and $x / \cosh ^{2}(x)$ are monotone and that $\tilde{U}$ is negative, it is easily seen that (27) is always $\leq 0$.

It is important to recall that, thanks to the presence of the pair-hopping term, an effective attractive interaction $\tilde{U}$ is consistent with a positive value of the Coulomb interaction $U$. Hence the present HF treatment of the thermodynamics of the PKH model yields a structured filling dependent superconducting phase even in presence of repulsive on site Coulomb interaction between electrons.

Finally, we wish to stress that the phase diagram in fig.8-obtained by including the next nearest neighbors hopping term- exhibits appealing features: for $\alpha \neq 0$ the optimal doping of the superconducting region is at $n_{\max } \neq 1$, and the critical temperature is enhanced. Moreover, with respect to the results reported in [16] on the 
attractive Hubbard model, our figure shows that even at $T=0$ the superconducting phase exists only for an appropriate range of filling values, not including half-filling. In fact, one could observe that the whole curve of the critical temperature vs. filling (8) is very reminiscent of that obtained for high- $T_{c}$ materials.

The study of the influence that particle-hole non symmetric terms in the Hamiltonian have on the features of the phase diagram has been worked out in 2- $d$, the conduction in high- $T_{c}$ superconducting materials tipically taking place along the cuprate planes. As Hartree-Fock approach is more accurate the higher is the dimension, dealing with a 3-d and anisotropic order parameter would possibly be more reliable. Work is in progress along these lines. At the same time, since the results obtained here are encouraging, a numerical study of the temperature behavior of the present model in $d=2$ would be probative. 


\section{REFERENCES}

[1] J. Hubbard, Proc. Roy. Soc. London, Sec. A 276, 238 (1963)

[2] R. Strack, D. Vollhardt, Phys. Rev. Lett. 72, 3425 (1994)

[3] A. Montorsi, and D.K. Campbell, Phys. Rev. B 53, 5153 (1996)

[4] F.H.L. Essler, V. Korepin, K. Schoutens, Phys. Rev. Lett. 68, 2960 (1992)

[5] F.H.L. Essler, V. Korepin, K. Schoutens, Phys. Rev. Lett. 70, 73 (1993)

[6] L. Arrachea, A.A. Aligia, Phys. Rev. Lett. 73, 2240 (1994)

[7] A. Schadschneider, Phys. Rev. B 51, 10386 (1995)

[8] J. de Boer, V. Korepin, A. Schadschneider, Phys. Rev. Lett. 74, 789 (1995)

[9] A.J. Bracken, M.D. Gould, J.R. Links, and Y-Z Zhang, Phys. Rev. Lett. 74, $2768(1995)$

[10] C.N. Yang, Rev. Mod. Phys. 34, 694 (1962); G.L. Sewell, J. Stat. Phys, 71, 415 (1990); H.T. Nieh, G. Su, B.-H. Zhao, Phys. Rev. B 51, 3760 (1995)

[11] Z. Szabó, Phys. Rev. B 59, 10007 (1999)

[12] M. Beccaria, Maximal Stability Regions for Superconducting Ground States of Generalized Hubbard Models, preprint cond-mat/9904128

[13] K.A. Penson, M.Kolb, Phys. Rev. B 33, 1663 (1986)

[14] A. Hui, S. Doniach, Phys. Rev. B 48, 2063 (1993)

[15] S. Robaszkiewicz, B.Bulka, Phys. Rev. B 59, 6430 (1999)

[16] R.Micnas, J.Ranninger, S. Robaszkiewicz, S. Tabor, Phys. Rev. B 37, 9410 (1988) 


\section{FIGURES}

FIG. 1. Phase diagram in $d=1$ at $T=0$ of AAS model (solid curve) and EKS model (dot-dashed curve). A filling-independent (superconducting) region is obtained in which the $\eta_{\alpha}$ pairs are ground state. Above it, a filling-dependent region (still superconducting) exists. In the EKS model the presence of a pair hopping term contributes to extend the superconducting zone towards positive values of $U$. In such exactly solved 1-d models the phase diagram reaches a maximum around half filling; moreover some unphysical constraint (for instance $X=t$ ) have to be imposed on the coupling constants in order to make the Hamiltonian integrable.

FIG. 2. The density of states in $d=3$ of the hopping term; notice that the value of

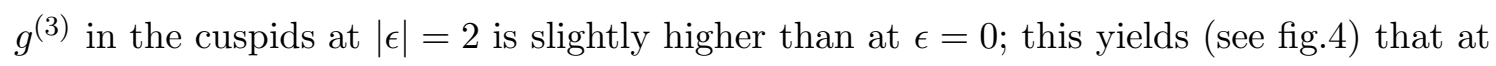
$T=0$ the maximum of the critical curve is reached away from half filling.

FIG. 3. Phase diagram of the $\pi$-phase in $d=2$; the critical value $\tilde{U}_{c}$ of the effective attraction $\tilde{U}=U-q Y$ is plotted as a function of the filling for some values of the

temperature $T$. At a given temperature the $\pi$-phase exists for $\tilde{U} \leq \tilde{U}_{c} ;$ here $\Delta=8 t$ is the bandwidth and $q=4$ is the number of nearest neighbors in the lattice. The values of $\tilde{U}$ are negative because the pair hopping term renormalizes the on-site Coulomb repulsion $U$ to a negative regime. Notice also that the curves are centered around half filling; at $T=0$ a filling independent region exists for $\tilde{U} \leq-\Delta$, like in AAS and EKS models (see fig.(1). 
FIG. 4. Phase diagram of the $\pi$-phase in $d=3 ; \Delta=12 t$ is the bandwidth and $q=6$ is the number of nearest neighbors in the lattice. With respect to the bidimensional case (see fig.(3) the curves have a plateau around half filling; indeed at $T=0$ the maximum values of $\tilde{U}_{c}$ are reached at the symmetric values $n \sim 0.4$ and $n \sim 1.6$. As the temperature is increased, the $\eta_{\pi}$-pairs become more likely to be broken and the extension of the $\pi$-phase reduces.

FIG. 5. The density of states in $d=2$ of the hopping term when a next nearest neighbors term of coupling constant $\alpha$ is also taken into account (solid line); notice the asymmetric behaviour with respect to the usual case $\alpha=0$ : the logarithmic divergence is shifted to $\epsilon=-4 \alpha$. This effect is due to the break up of the particle-hole invariance of the hopping term; it yields an asymmetric shape of the phase diagram (see fig.66).

FIG. 6 . The $\pi$-phase diagram in $d=2$ for different values of the next-nearest neighbors hopping amplitude $\alpha$ at the temperature $k_{B} T / t=0.1$. Notice that when $\alpha$ is increased the superconducting region rises up towards less negative values of the $\tilde{U}=U-q Y$, and its maximum is reached at a $n_{\max }$ which moves away from half filling. This means that, at a given temperature, the next-nearest neighbors interaction reduces the effective attraction $\tilde{U}$, yielding an increase of the highest reachable critical temperature (see also fig.8).

FIG. 7. The behaviour of $n_{\max }$ (i.e. the filling at which the critical curve $\tilde{U}_{c}$ vs $n$ in fig.6 reaches the maximum) as a function of the next nearest neighbors coupling constant $\alpha$ (in units of $t$ ), at temperature $k_{B} T / t=0.1$. The curve is odd. Notice that for $-0.4 \leq \alpha \leq 0.4$ the behaviour is almost linear; as $|\alpha|$ is furtherly increased, $n_{\max }$ saturates to 0 or to 2 , as one can also see in fig.6. It is possible to show that this behaviour of $n_{\max }$ depends very weakly on the temperature $T$. 
FIG. 8. The critical temperature as a function of the filling in $d=2$, for a given value $\tilde{U}=-4 t$ of the effective attractive interaction. Here $\alpha$ is the coupling constant of the next nearest neighbors hopping term, which breaks the particle-hole symmetry of the model. Notice that, with respect to the case $\alpha=0$, the next nearest neighbors term yields both the increase of the highest critical temperature and the displacement away from half filling of the superconducting $\pi$-phase. In fact the highest $T_{c}$ is reached at $n \sim 1.3$. 


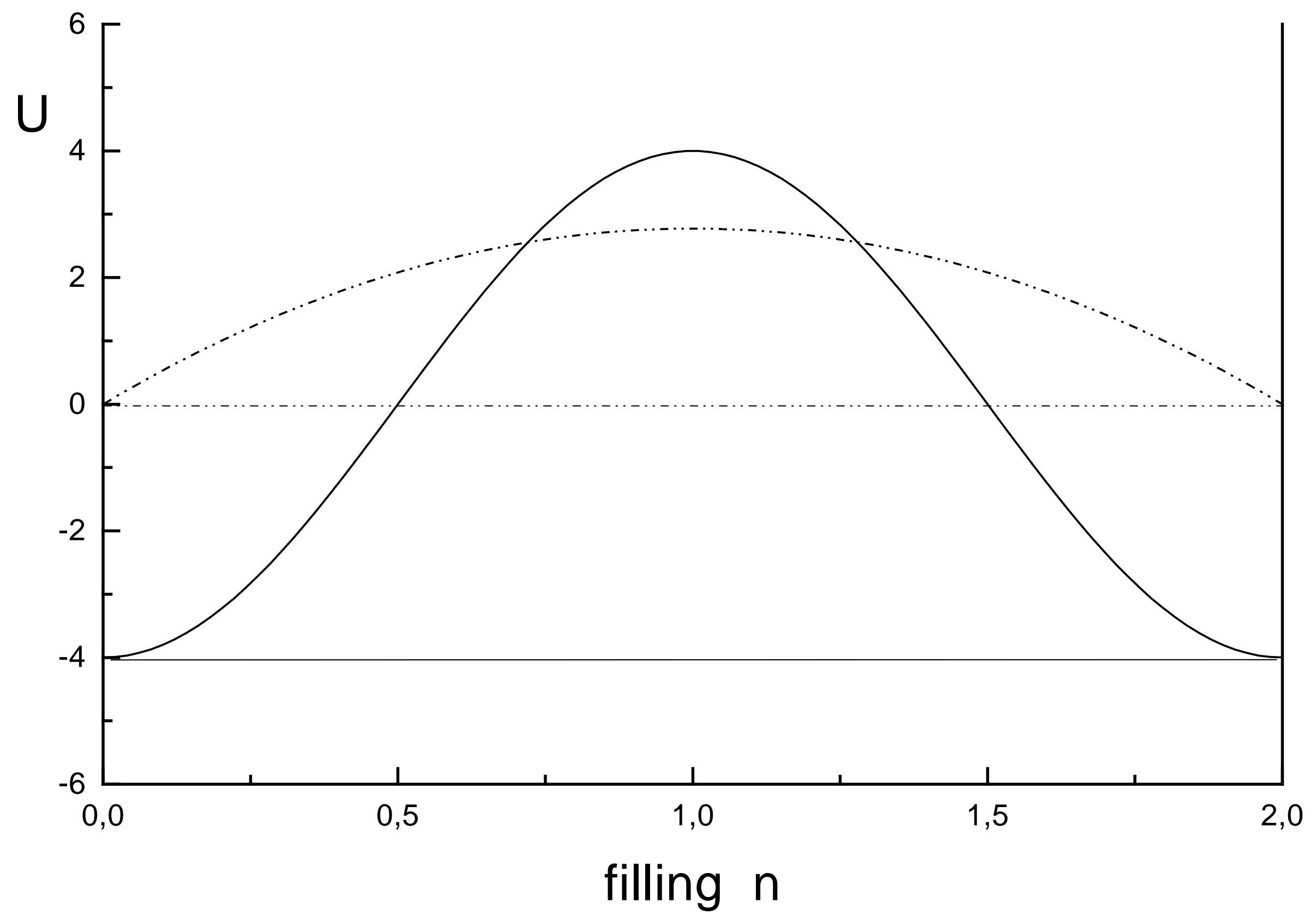




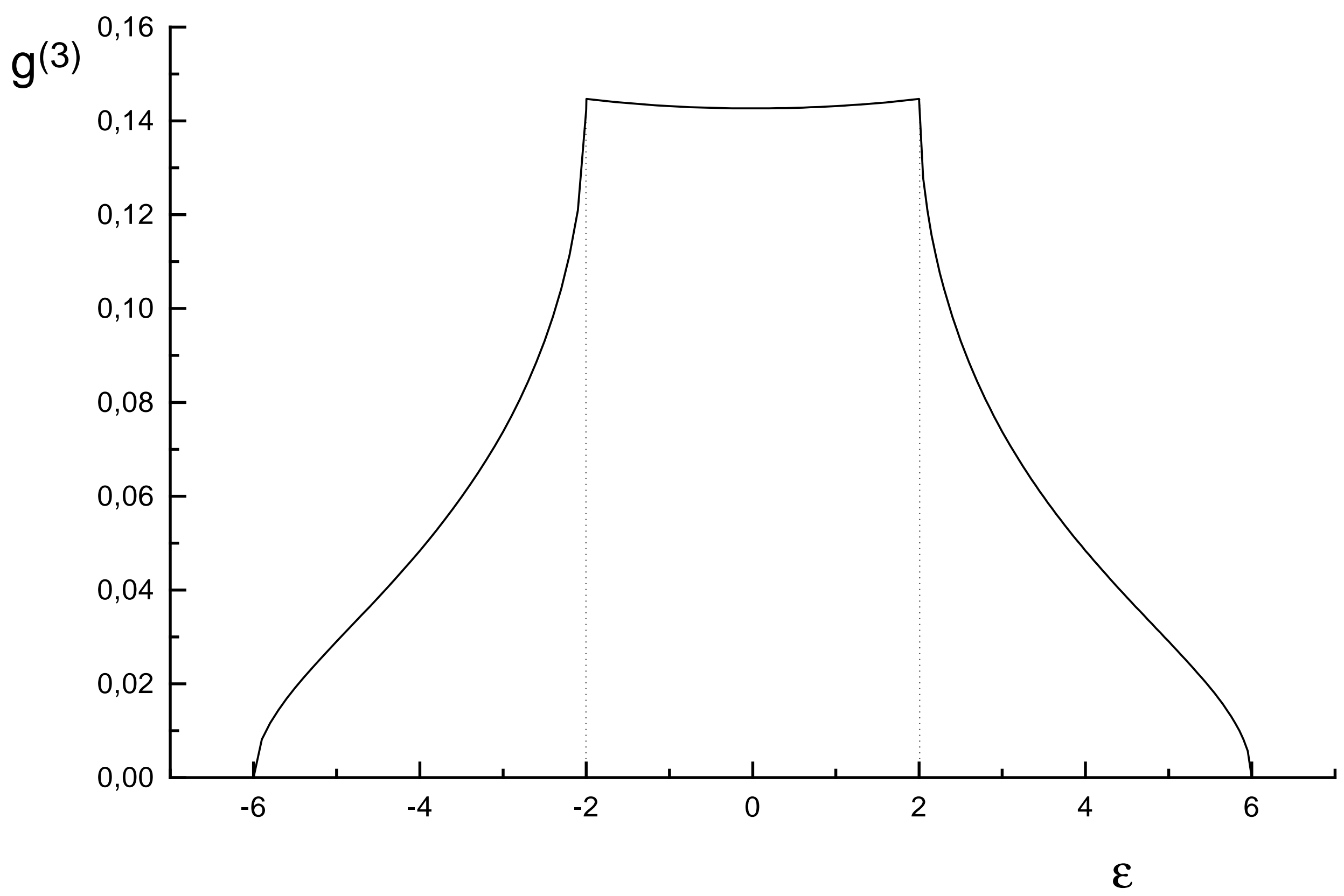




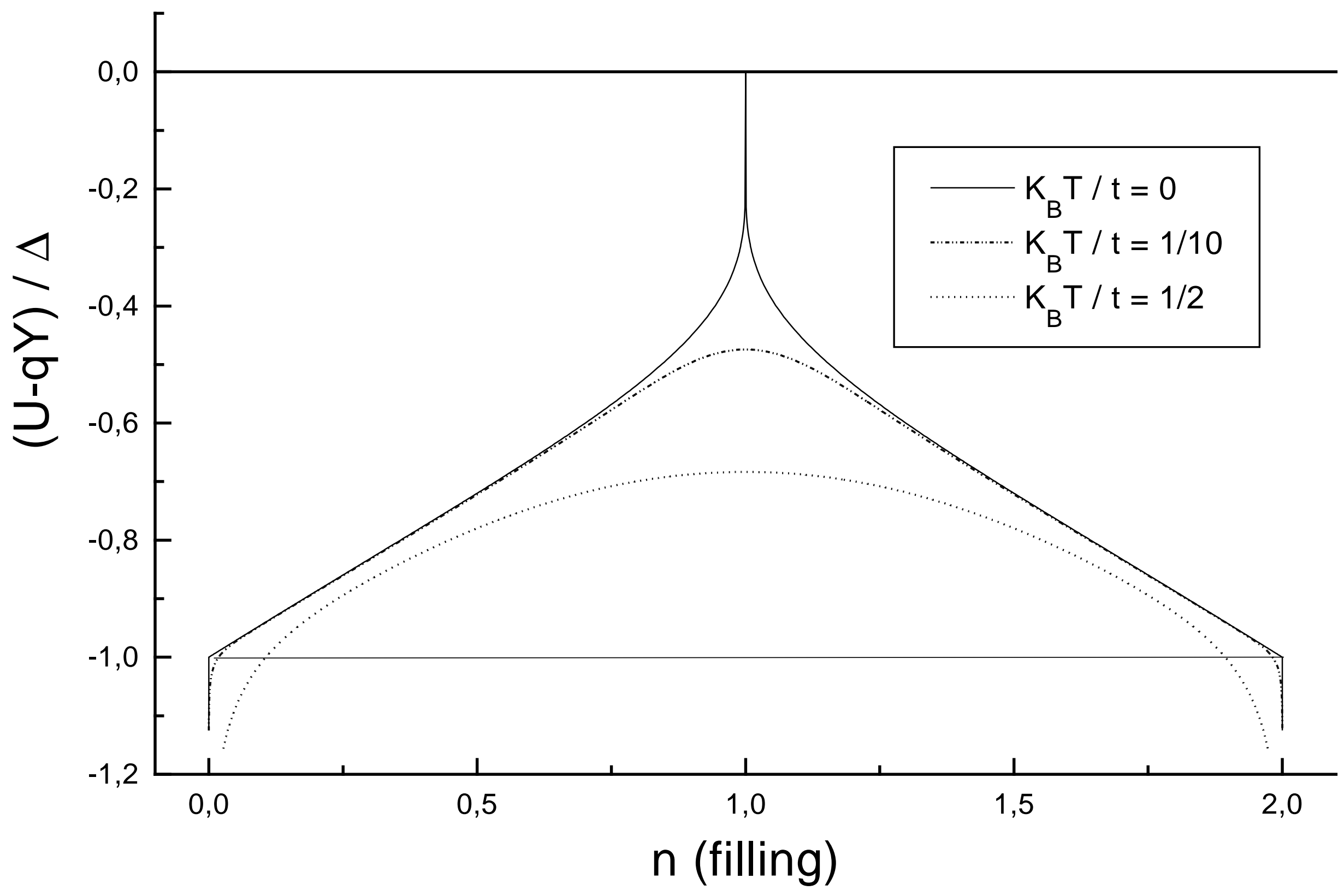




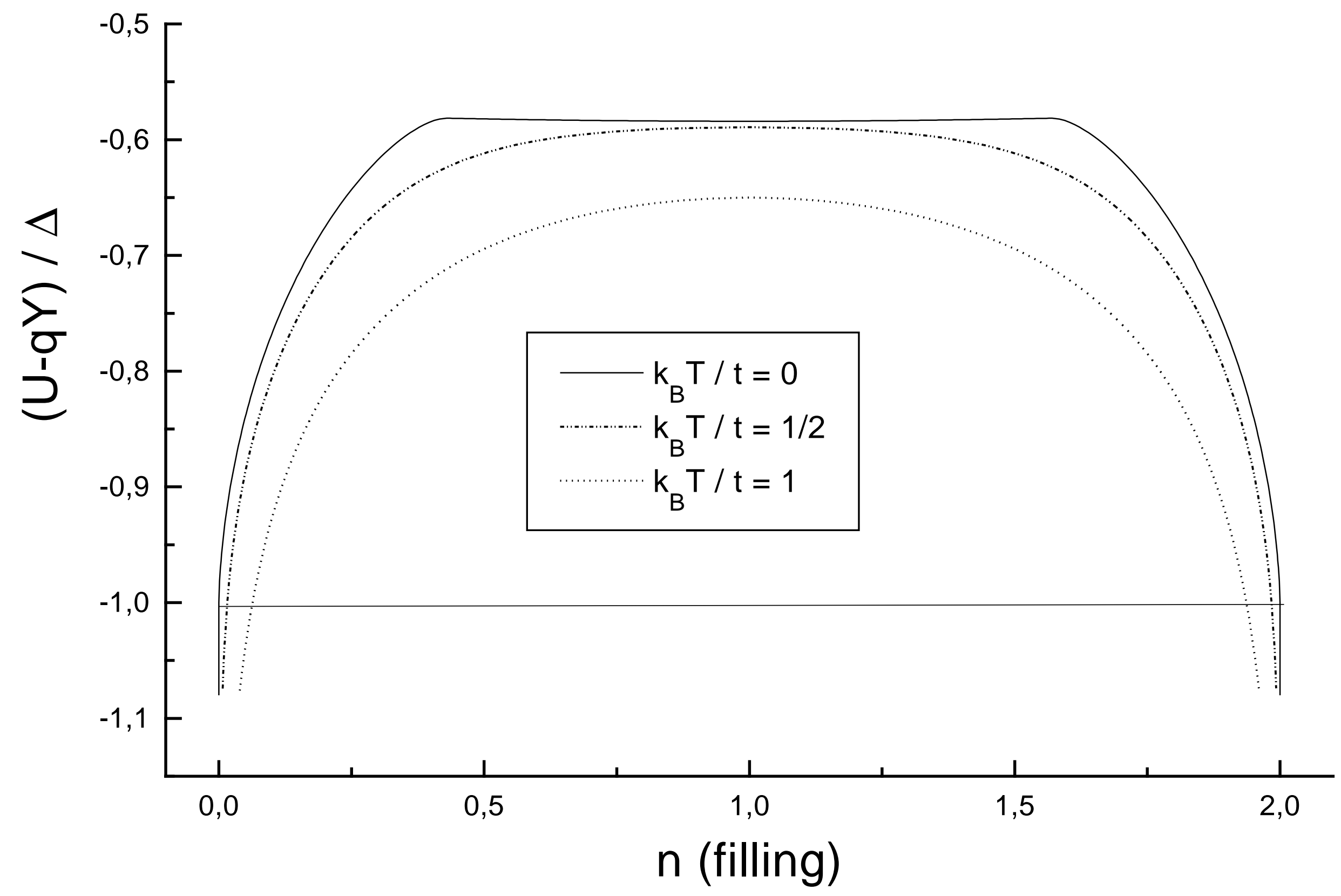




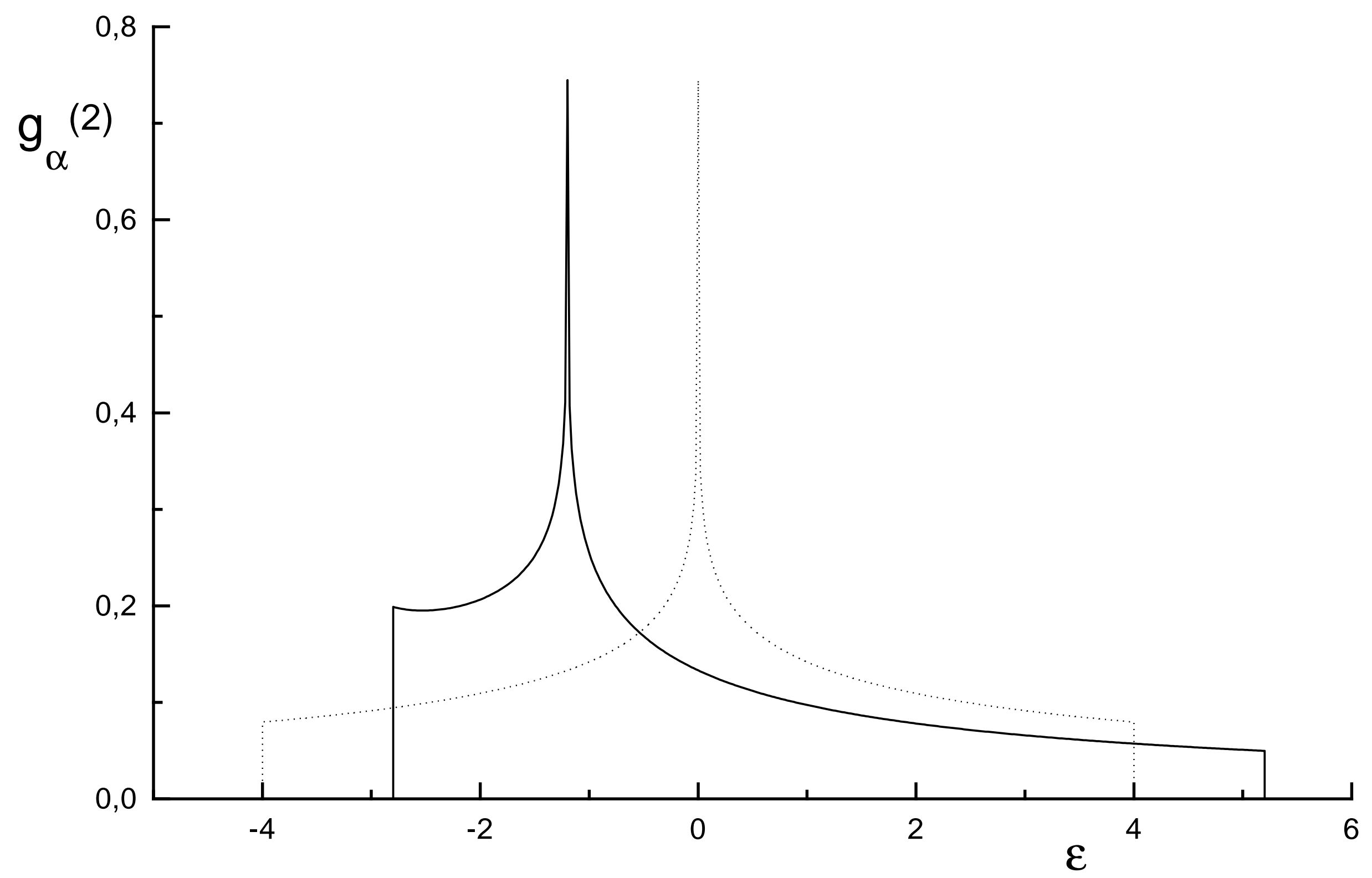




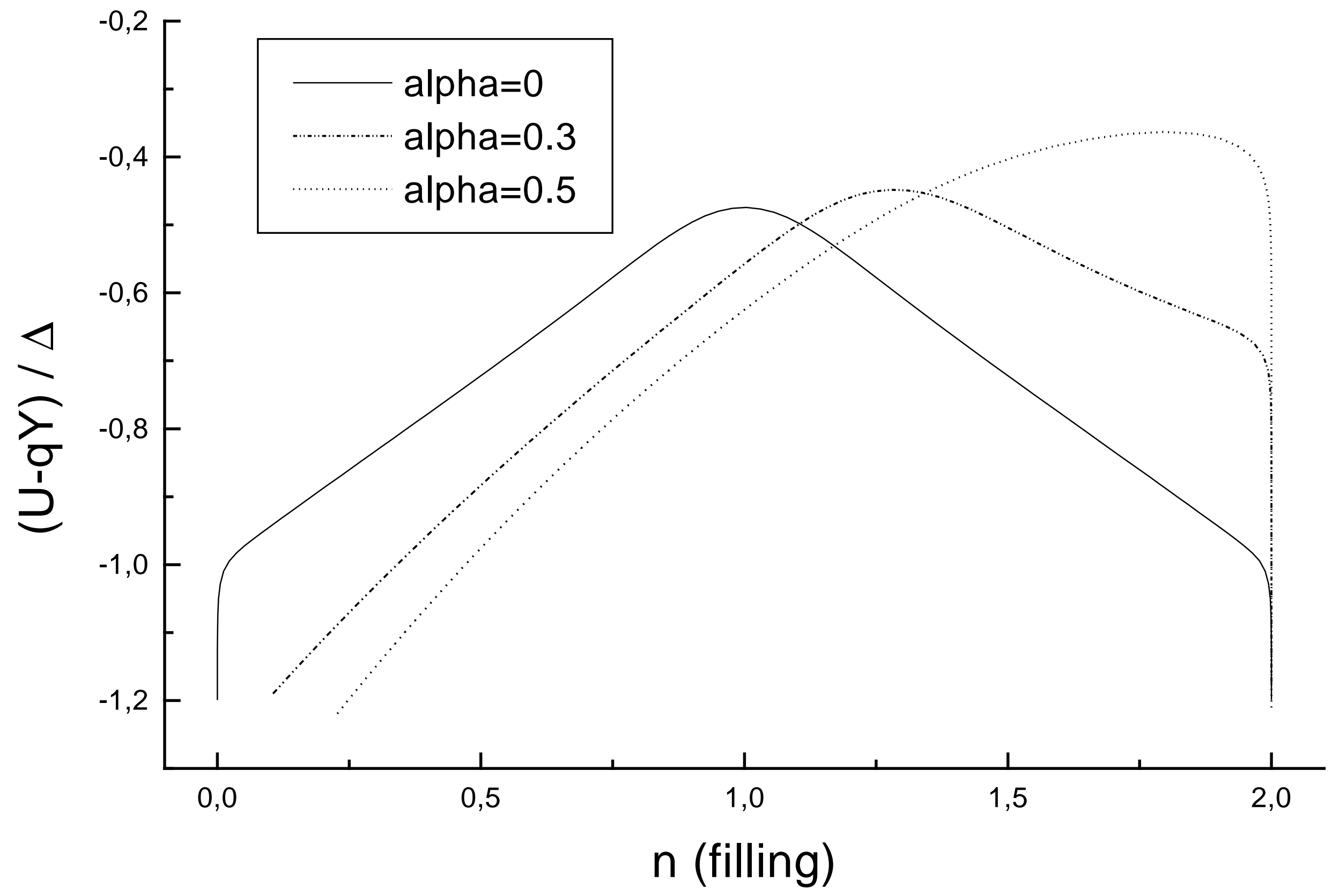




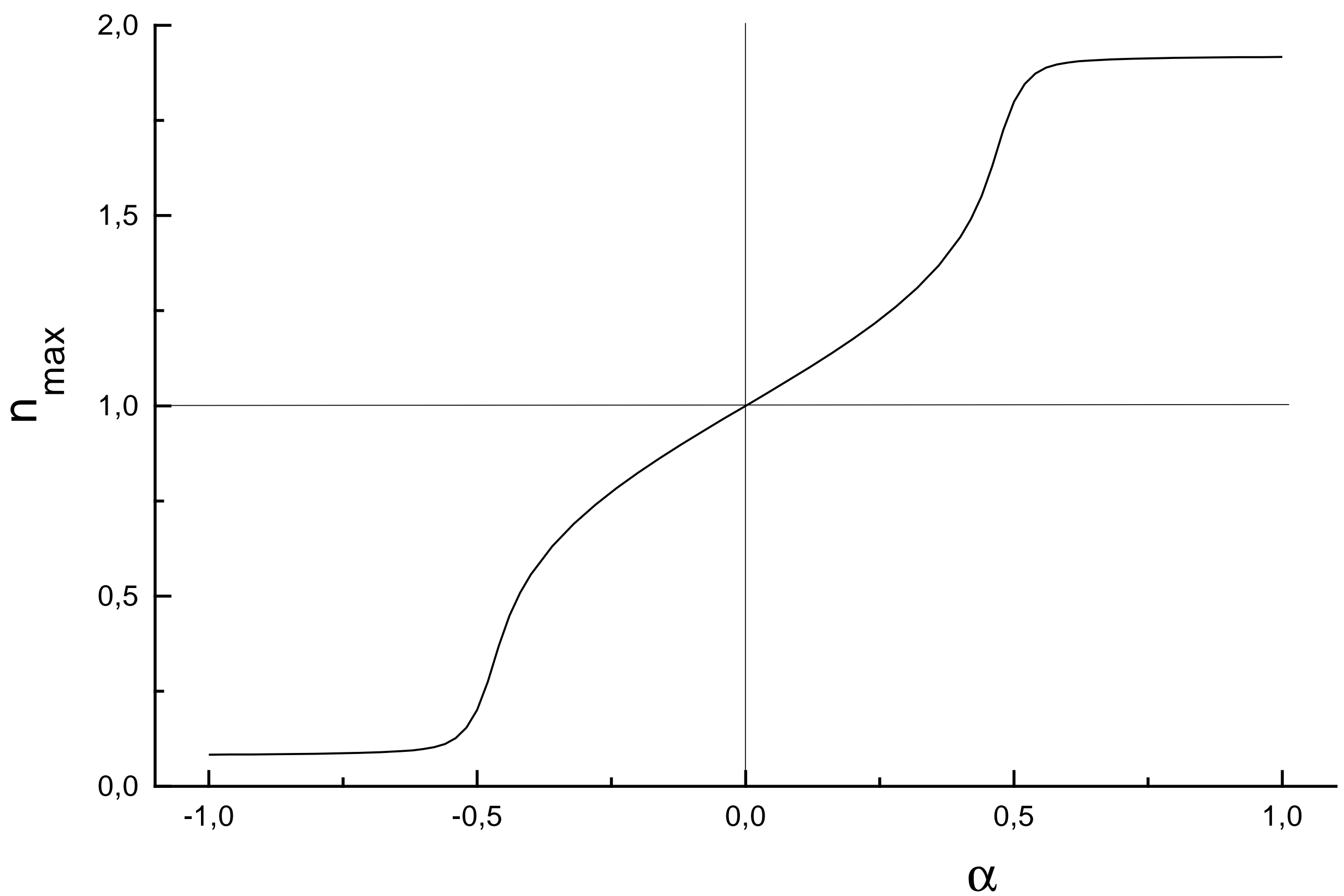




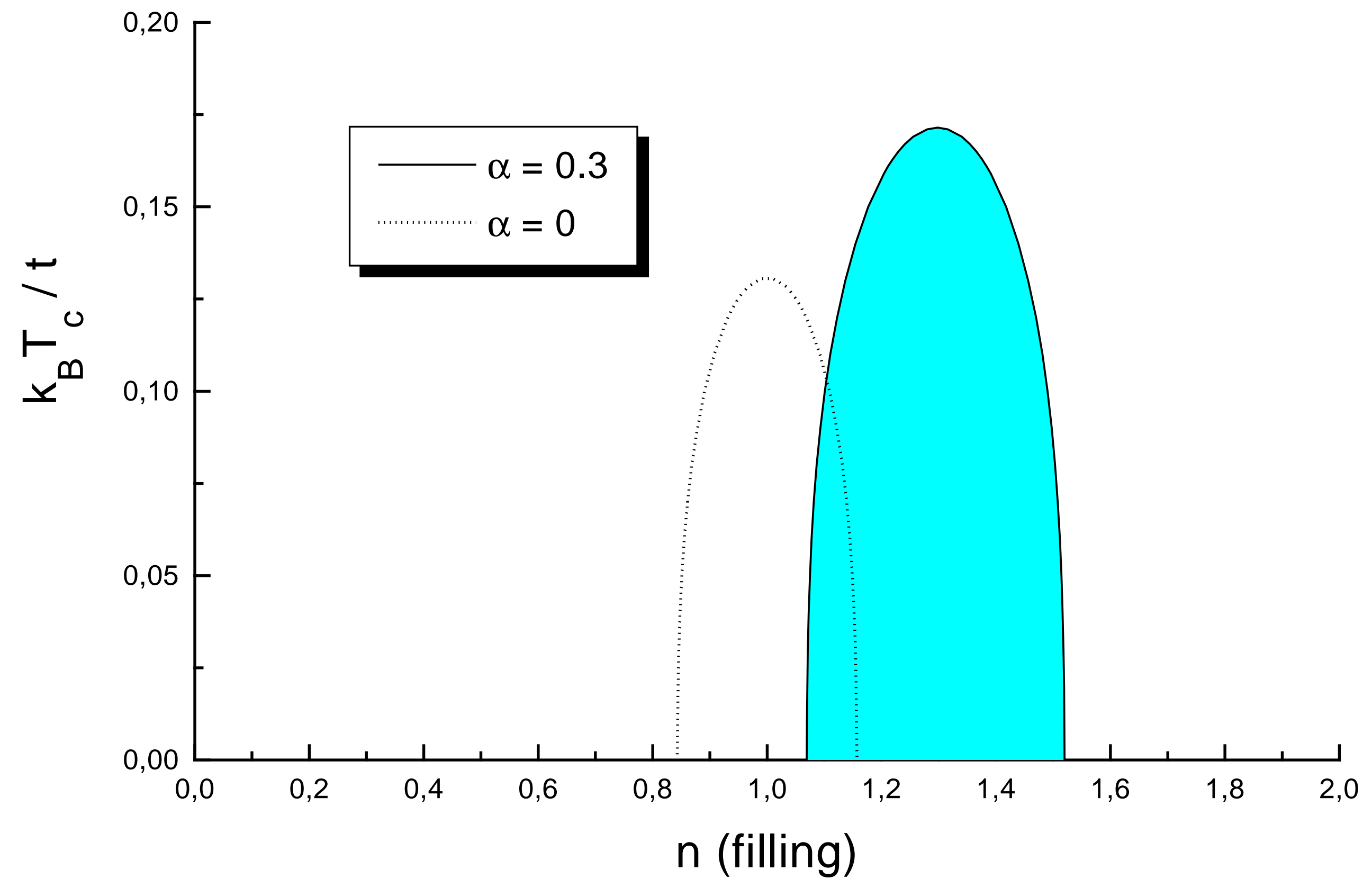

